\title{
Top quark physics in ATLAS
}

\author{
Carolina Gabaldon*, on behalf of the ATLAS Collaboration \\ LPSC, Université Grenoble-Alpes, CNRS/IN2P3 \\ 53, rue des Martyrs 38026 Grenoble Cedex \\ *E-mail: carolina@cern.ch
}

\begin{abstract}
This paper summarises the latest experimental results on top quark physics by the ATLAS Collaboration using LHC $p p$ collisions data corresponding to an integrated luminosity of $4.7 \mathrm{fb}^{-1}$ at $\sqrt{s}=7 \mathrm{TeV}$ and $20.3 \mathrm{fb}^{-1}$ at $\sqrt{s}=8 \mathrm{TeV}$. Results presented here cover different aspects of the top quark studies including searches for physics beyond the Standard Model.
\end{abstract}

Keywords: LHC, ATLAS, top quark

\section{Introduction}

Since the top quark was discovered at the Tevatron in 1995, it has remained a major focus for particle physics research. This interest comes partly from its large mass ${ }^{1}$, $173.34 \pm 0.76 \mathrm{GeV}$, close to the electroweak symmetry breaking (EWSB) scale which suggests that searches involving the top quark or some of its possible partners could provide non-trivial information on the EWSB sector. Moreover, the lifetime of the top quark is shorter than the hadronisation time scale, thus it decays almost $100 \%$ of the time to a $W$ boson and a $b$-quark. For these reasons, research in top quark physics could serve as a window to new physics beyond the Standard Model (SM), and precise measurements of the properties of the top quark and its interactions are of crucial importance for a better understanding of the SM predictions.

In its first years of operation, the LHC has proven to be a top quark factory, allowing ATLAS ${ }^{2}$ and CMS to study many aspects of this particle with great accuracy and to search for new physics involving the top quark. This article presents a few recent ATLAS results involving top quarks. The full listing of top quark measurements by the ATLAS experiment can be found in Ref. 3 .

\section{Top quark production}

At the LHC, the SM top quarks are produced in pairs via the strong interaction or singly via the weak interaction. Top pair production is the dominant mechanism at the LHC and is described in terms of quantum chromodynamics (QCD). Single top quarks are produced through a $W t b$ vertex via three modes: the exchange of a virtual $W$ boson in the $t$-channel or in the $s$-channel and the associated production of a top quark with an on-shell $W$ boson. 


\subsection{Inclusive cross-section measurements}

The $t \bar{t}$ cross-sections measured at $7-8 \mathrm{TeV}$ by ATLAS are summarised in Figure 1. All numbers are consistent within uncertainties with the SM expectation at the nextto-next-to-leading order (NNLO) complemented with the next-to-next-to-leading logarithmic (NNLL) resummation.

At $8 \mathrm{TeV}$, the $t \bar{t}$ cross-section has been measured in the lepton+jets ${ }^{4}$ and dilepton ${ }^{5}$ channels. The dilepton channel gives the most precise ATLAS $t \bar{t}$ cross-section measurement, selecting opposite-charge electron-muon pairs in the final state. In this analysis, events with exactly one and two b-tagged jets are counted and used to determine simultaneously the $t \bar{t}$ cross section and the efficiency to reconstruct and tag a $b$-jet from the top quark decay. A cross-section of $\sigma_{t \bar{t}}=237.7 \pm 1.7$ (stat.) \pm 7.4 (syst.) \pm 7.4 (lumi.) \pm 4.0 (beam energy) pb is extracted with a total relative uncertainty of $4.8 \%$.

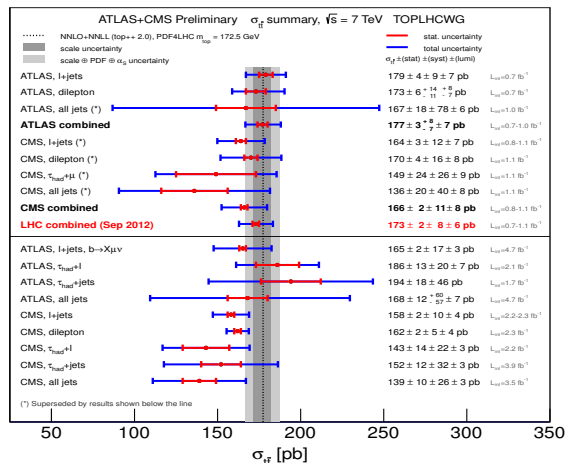

(a)

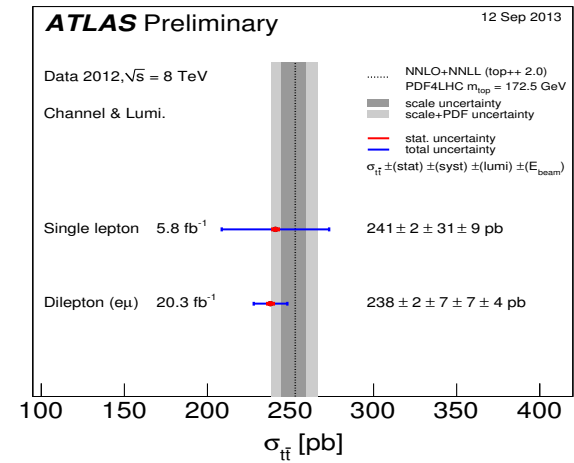

(b)

Fig. 1: (a) The ATLAS $\sigma_{t \bar{t}}$ measurements ${ }^{3}$ in all decay modes and the LHC combination at $7 \mathrm{TeV}$ and (b) the ATLAS recent measurements ${ }^{3}$ at $8 \mathrm{TeV}$.

Figure 2 (a) summarises the single top cross-section measurements performed with the 2011 and 2012 datasets. All results are consistent with the NLO+NNLL SM expectations. The process with the highest expected cross section at the LHC is the $t$-channel mode. The analysis ${ }^{6}$ at $8 \mathrm{TeV}$ used a new approach that allow to extract the production cross-section from the measurement of the $t$-channel fiducial cross-section. In this way, the theoretical modelling uncertainty is minimised. A Neural Network (NN) classifier is used to determine the cross-section in a fiducial volume within the detector acceptance. The fiducial cross-section is measured as $\sigma_{\text {fid }}=3.37 \pm 0.05$ (stat.) \pm 0.47 (syst.) \pm 0.09 (lumi) pb. Figure 2 (b) shows the comparison with different generators. The acceptance difference between generators is as large as the theoretical uncertainties. A cross-section of $\sigma_{t \text {-channel }}=82.6 \pm$ 1.2 (stat.) \pm 11.4 (syst.) \pm 3.1 (PDF) \pm 2.3 (lumi.) pb is extracted when extrapolated to the full phase space. The total uncertainty is $17 \%$ compared to $14 \%$ for the fiducial cross-section. 
Due to its relatively small cross-section, the $W t$-channel is detected for the first time at the LHC. A measurement is performed at $8 \mathrm{TeV}$ using dilepton final states ${ }^{7}$. A Boosted Decision Tree (BDT) technique is used to separate $W t$-channel events from the expected background. The two BDT classifiers (1 and 2 jets regions) are fitted by simultaneous maximum likelihood to extract the cross-section $\sigma_{W t}=$ $27.2 \pm 2.8$ (stat.) \pm 5.4 (syst.) pb with an observed significance of $4.2 \sigma$.

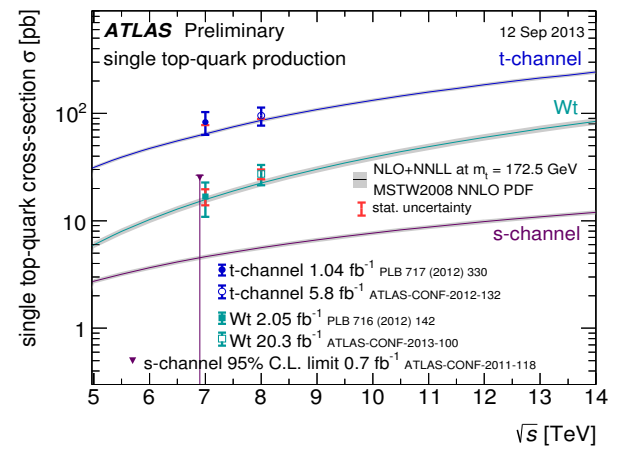

(a)

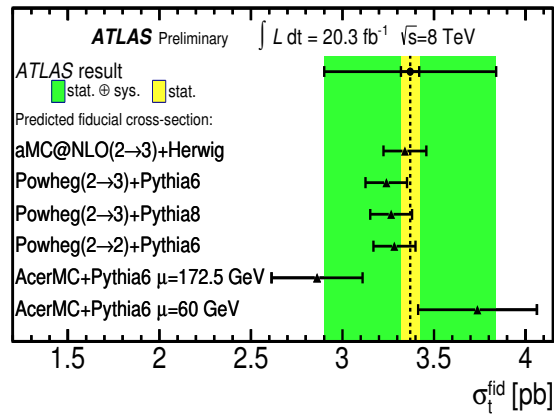

(b)

Fig. 2: (a) Summary of the ATLAS single top cross-section measurements ${ }^{3}$ at 7 and $8 \mathrm{TeV}$. (b) The $t$-channel fiducial cross-section measurement compared with NLO and LO predictions ${ }^{6}$.

\subsection{Differential cross-section measurements at $\sqrt{s}=7 \mathrm{TeV}$}

Since predictions and measurements for the $t \bar{t}$ cross-section are in good agreement at the same precision level, the differential measurements are starting to play an important role to test the theoretical QCD calculations. ATLAS performed an analysis to measure the relative $t \bar{t}$ cross-section as a function of several kinematic variables with the full $7 \mathrm{TeV}$ dataset $^{8}$. The reconstruction of the kinematic distributions is performed through a kinematic fit using a maximum likelihood approach. Afterwards, the data are unfolded via a regularised Singular Value Decomposition (SVD) method. The measurements are compared to various MC generators, theoretical predictions and PDF sets. When comparing the unfolded data to the MC generators, the top quark transverse momentum $\left(p_{T}^{t}\right)$ distribution (see Fig. 3 (a)) shows a large deviation between the data and Alpgen at large values of $p_{T}^{t}$. In Figure 3 (b) several PDF sets are compared with the $t \bar{t}$ mass $\left(m_{t \bar{t}}\right)$ distribution. Here, the HERAPDF set gives the best data description.

\section{Top quark mass measurements}

LHC measurements of the top quark mass in several channels are combined with the results obtained at the Tevatron, as summarised in Fig. 4 (a). The combination ${ }^{9}$ is performed using the Best Linear Unbiased Estimate (BLUE) method. The world combination result, $173.34 \pm 0.76 \mathrm{GeV}$, has a total relative uncertainty of $0.4 \%$. 


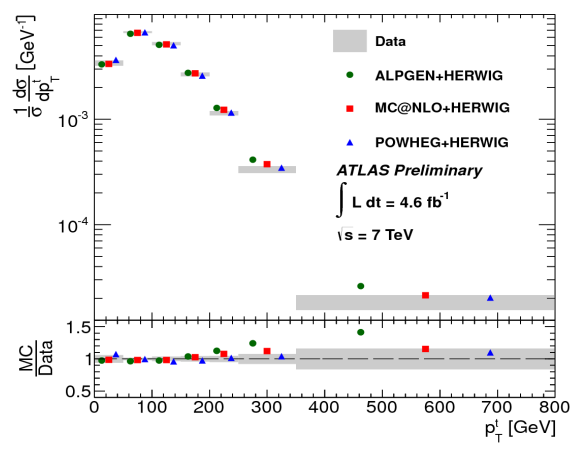

(a)

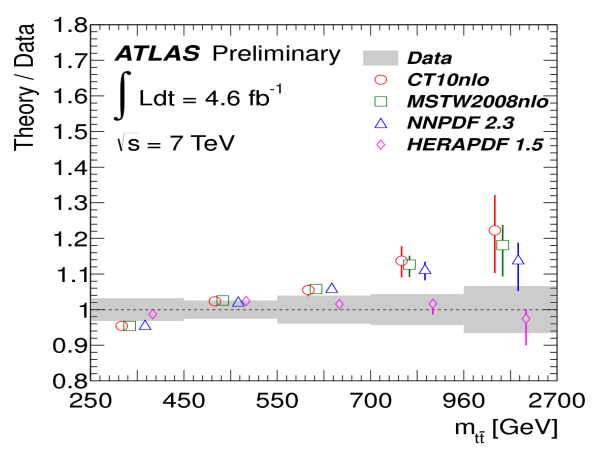

(b)

Fig. 3: (a) Normalised differential cross-sections for the transverse momentum of the hadronically decaying top quark ${ }^{8}$ and (b) the mass of the $t \bar{t}$ system $^{8}$.

The most precise ATLAS mass measurement is obtained with a threedimensional fit in the lepton + jets channel ${ }^{10}$. Three observables are reconstructed for the selected events, the top mass, the mass of the $W$ boson and $R_{l b}$ defined as the ratio between the transverse momenta of $b$-jets and light-jets in the hadronic decay of the top quark. A large number of templates are generated for various top mass hypotheses in the experimentally allowed range, and different energy-scale factors of light jets (JSF) and $b$-jets (bJSF). An unbinned 3-dimensional fit results in a mass measurement of $m_{\text {top }}=172.31 \pm 0.75$ (stat. $\left.+\mathrm{JSF}+\mathrm{bJSF}\right) \pm 1.35$ (syst.) $\mathrm{GeV}$ with a total uncertainty less than $1 \%$.

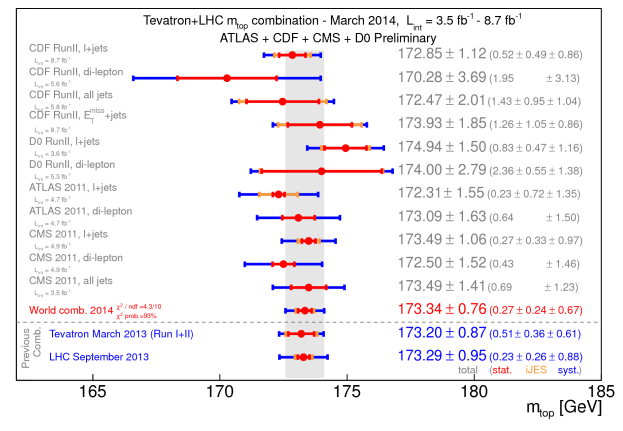

(a)

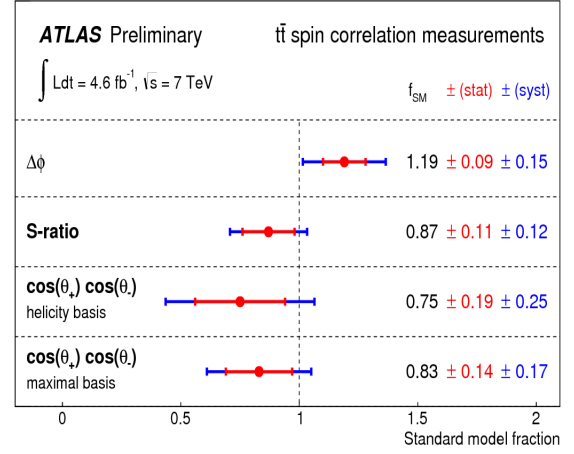

(b)

Fig. 4: (a) Top mass measurements summary for LHC and Tevatron and the world mass combination ${ }^{9}$. (b) Summary of the measurements of $f_{S M}$ using four spin correlation observables ${ }^{10}$.

\section{Top quark properties}

The ATLAS experiment measured many of the top quark properties with unprecedented precision, offering both stringent tests of the SM and possible pathways to discovering new physics. 
The top quark decays before hadronisation, which allows a study of the spin correlation in the $t \bar{t}$ system via the angular distributions of its decay products. An analysis is carried out by ATLAS using the $t \bar{t}$ events collected at $7 \mathrm{TeV}$ in the dilepton topology ${ }^{11}$. Four observables are measured to extract the spin correlation strength $\left(f_{S M}\right)$ : the azimuthal angle between the two charged leptons $(\Delta \phi)$, the ratio of the correlated and the uncorrelated spin matrix elements $(S-$ ratio $)$ and the $\cos \left(\theta_{+}\right) \cos \left(\theta_{-}\right)$in the helicity and maximal basis. The results are extracted with a binned maximum likelihood fit to the four distributions (see Fig. 4 (b)). All measurements agree with the SM prediction of $f_{S M}=1$.

Another interesting top quark property is the $t \bar{t}$ production charge asymmetry $\left(A_{c}\right)$. These measurements not only provide a possibility to test QCD at high energies but also is an ideal place to observe effects coming from BSM processes. ATLAS performed at $7 \mathrm{TeV}$ an analysis ${ }^{12}$ using the lepton+jets topology. This study used the differential distributions of several observables $\left(p_{T}\right.$, mass and rapidity) of the $t \bar{t}$ system to extract $A_{c}$. In order to compare with the theoretical predictions, these distributions are unfolded to correct for event selection bias and detector effects. The measured value of the charge asymmetry is $A_{C}=0.006 \pm 0.010$. The ATLAS results are combined with the CMS ones in Ref. 13. The LHC combination $\left(A_{C}=0.005 \pm 0.009\right)$ is in agreement with the NLO SM prediction.

\section{Beyond Standard Model searches with top quarks}

Due to its heavy mass, top quarks are often expected between the decay products of heavy BSM particles. Among the possible new couplings, the Flavour Changing Neutral Currents (FCNCs) are one of the most interesting processes. It is highly suppressed in the SM, but several BSM scenarios predict enhanced FCNC rates. A search for $t$-channel production via $\mathrm{FCNC}^{14}, q g \rightarrow t \rightarrow l \nu b$, is carried out using $14.2 \mathrm{fb}^{-1}$ ATLAS data at $8 \mathrm{TeV}$. The same analysis strategy than in the $t$-channel analysis is used. A NN technique is employed to separate the FCNC events from the SM background and then, the NN classifier is fitted using a Bayesian approach. No deviation from SM predictions are found, therefore limits are set on the branching ratios at $95 \%$ C.L.: $B(t \rightarrow c g)<1.6 \cdot 10^{-4}, B(t \rightarrow u g)<3.1 \cdot 10^{-5}$ (see Fig. 5(a)).

Another interesting topic is the search for $t \bar{t}$ resonances. Using $8 \mathrm{TeV}$ data $\left(14.2 \mathrm{fb}^{-1}\right)$, ATLAS searched for the production of these resonances in the lepton + jets topology ${ }^{15}$. The $t \bar{t}$ invariant mass spectrum is tested for any excess of events that could imply a $t \bar{t}$ resonance coming from a new heavy particle such as the $Z^{\prime}$ boson. In the high-mass regime, event topologies can change and top quark decay products become highly boosted, requiring the use of special identification algorithms. To reconstruct the $m_{t \bar{t}}$ distribution a combination of resolved and boosted methods are used. No evidence of a resonance is found. Limits are set on the production cross-section times branching ratio to $t \bar{t}$ for narrow and wide resonances. Figure 5 (b) shows the observed and expected upper cross-section limits on $Z^{\prime}$ bosons. ATLAS excludes $Z^{\prime}$ bosons with masses up to $1.8 \mathrm{TeV}$. 


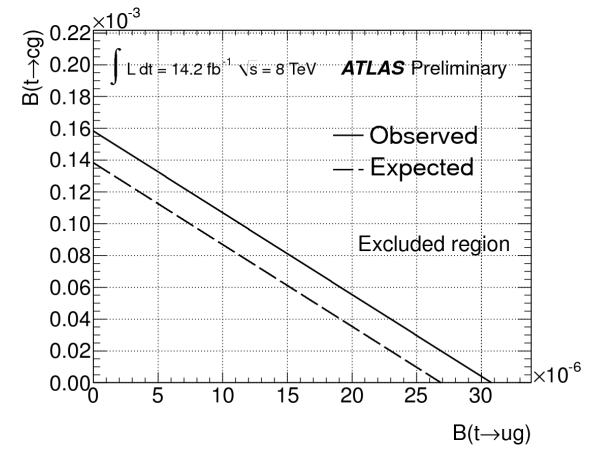

(a)

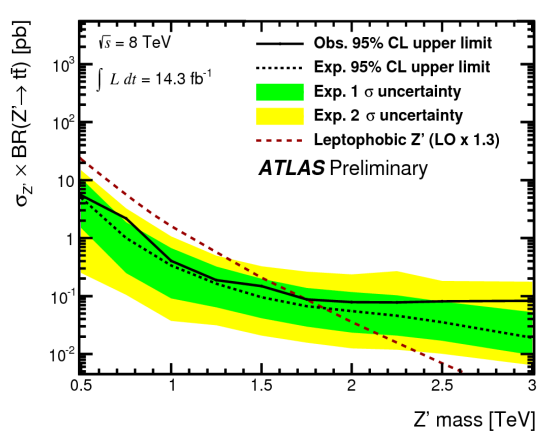

(b)

Fig. 5: (a) Upper limits on the branching fractions for the FCNC processes ${ }^{14}$. (b) Upper limits on a narrow-width $Z^{\prime}$ boson ${ }^{15}$.

\section{Conclusions}

With the excellent performance of the LHC, the experimental physics of the top quark entered in a precision era with all measurements limited by systematic uncertainties. The $t \bar{t}$ cross-section has been measured with a precision better than $5 \%$ and the mass of the top with a precision of about $1 \%$. A large variety of top quark properties in top quark pair production and decay have been studied. Though, within uncertainties, no signs of BSM physics have been observed, these measurements have significantly extended the region of validity of the SM and have found new exclusion limits on a variety of the BSM scenarios.

\section{References}

1. ATLAS, CDF, CMS, D0 Collaborations, arXiv:1403.4427 [hep-ex].

2. ATLAS Collaboration, 2008 JINST 3 S08003.

3. https://twiki.cern.ch/twiki/bin/view/AtlasPublic/TopPublicResults.

4. ATLAS Collaboration, ATLAS-CONF-2012-149, https://cds.cern.ch/record/1493488.

5. ATLAS Collaboration, ATLAS-CONF-2013-097, https://cds.cern.ch/record/1600596.

6. ATLAS Collaboration, ATLAS-CONF-2014-007, https://cds.cern.ch/record/1668960.

7. ATLAS Collaboration, ATLAS-CONF-2013-100, https://cds.cern.ch/record/1600799.

8. ATLAS Collaboration, ATLAS-CONF-2013-099, https://cds.cern.ch/record/1600778.

9. ATLAS, CDF, CMS and DO Collaborations, arXiv:1403.4427 [hep-ex]

10. ATLAS Collaboration, ATLAS-CONF-2013-046, https://cds.cern.ch/record/1547327.

11. ATLAS Collaboration, ATLAS-CONF-2013-101, https://cds.cern.ch/record/1600803.

12. ATLAS Collaboration, JHEP02 (2014) 107.

13. ATLAS Collaboration, ATLAS-CONF-2014-012, https://cds.cern.ch/record/1670535.

14. ATLAS Collaboration, ATLAS-CONF-2013-063, https://cds.cern.ch/record/1562777.

15. ATLAS Collaboration, ATLAS-CONF-2013-052, https://cds.cern.ch/record/1547568. 